\title{
L'altérité de l'altérité ou la question des sentiments en anthropologie
}

Monique Jeudy-Ballini

\section{OpenEdition}

1 Journals

Édition électronique

URL : http://journals.openedition.org/jso/6035

DOI : $10.4000 /$ jso.6035

ISSN : 1760-7256

Éditeur

Société des océanistes

Édition imprimée

Date de publication : 15 décembre 2010

Pagination : 129-138

ISBN : 978-2-85430-027-7

ISSN : 0300-953x

Référence électronique

Monique Jeudy-Ballini, «L'altérité de l'altérité ou la question des sentiments en anthropologie », Journal de la Société des Océanistes [En ligne], 130-131 | 2010, mis en ligne le 15 décembre 2013, consulté le 09 juin 2020. URL : http://journals.openedition.org/jso/6035 ; DOI : https://doi.org/ $10.4000 /$ jso. 6035 


\title{
L'altérité de l'altérité ou la question des sentiments en anthropologie
}

\author{
par
}

Monique JEUDY-BALLINI*

\section{RÉSUMÉ}

Comment approcher ethnographiquement les manières spécifiques de sentir dans les sociétés qu'on étudie? La relégation relative dans laquelle l'anthropologie océaniste contemporaine tient la question des sentiments peut renvoyer à l'opacité qui entoure l'accès aux affects d'autrui dans certaines communautés. Mais elle renvoie surtout, semble-t-il, à la tendance à exclure l'analyse des sentiments du champ des objets ethnographiables, ou à ne les aborder que de manière anecdotique. Sans doute cela s'explique-t-il par l'idée qu'on se fait de communautés attachées au respect de la tradition et au sein desquelles les comportements supposeraient une forte dose de normalisation collective; ou encore par l'idée qu'il n'est pas utile de s'attarder sur des ressentis censés relever d'expériences universelles (l'affliction, la joie, la honte, la douleur, etc. ). Dans la mesure, toutefois, où l'on ignore a priori ce qui se définit comme émotion dans les sociétés qu'on se propose d'étudier et dans la mesure aussi où maintes cultures ne font pas de différence entre affect et cognition, on se gardera de parler ici d'anthropologie des émotions en insistant simplement sur la nécessité de penser ensemble des dimensions indissociables dans toute expérience sociale.

MoTS-CLÉS : affects, bonheur, cognition, émotions, esthétique, guerre, incroyance, sentiments, sensations, violence

Dans les jours qui précédèrent la remise à l'éditeur du manuscrit de l'ouvrage collectif $P e o$ ple and Things que Bernard Juillerat et moi co-dirigions (2002), nos ultimes interventions concernèrent l'achèvement d'un index général. Alors que ce dernier était quasiment au point, je

\section{ABSTRACT}

How might particular ways of feeling in different societies be approached ethnographically? The relative lack of interest in feeling in contemporary Oceanic anthropology may simply be an indication of the opacity hindering ethnographic attempts to access the realm of affects in certain communities. Yet it appears primarily to reflect a tendency in contemporary anthropology to exclude the analysis of feelings from the field of objects potentially subject to ethnographic research, or to address such objects merely anecdotally. This can no doubt be explained by received views of communitiesthat value the respect of tradition and in which behaviours presuppose a high degree of collective normalization. It could also be explained by the view that there is little point in studying feelings deemed to reflect universal experiences (such as affliction, joy, shame, pain, etc.). Yet since we do not know what counts as an emotion in societies subject to ethnographic research, and since many cultures draw no rigid distinction between affect and cognition, this paper will make no reference to the anthropology of emotions and will insist simply on the need for a holistic consideration of factors deemed to be indissociable in any social experience.

KeYwORDS: affects, happiness, cognition, emotions, aesthetic, war, unbelief, feelings, sensations, violence

réalisai que nous n'avions pas prévu d'entrée pour la notion d'émotions ou d'affects. Quand je suggérai d'en ajouter une, Bernard ne trouva pas l'idée très pertinente au regard de la problématique générale du livre. Dans l'étude des médiations sociales en Océanie, en effet, la question des

* CNRS LAS, m.jeudy-ballini@college-de-france.fr 
émotions n'occupait à ses yeux qu'une place périphérique et ses relectures attentives des contributions que nous avions réunies sur la Mélanésie, la Polynésie et l'Australie ne l'avaient pas amené à se faire une opinion différente. À l'évidence, mes arguments ne le convainquirent pas davantage alors que, rétrospectivement $\mathrm{du}$ moins, j'ai le sentiment d'avoir été plutôt insistante. Il réagit cependant comme il l'avait toujours fait avant, durant et bien après les mois de notre collaboration, c'est-à-dire de manière bienveillante et presque avec amusement, sans chercher à tout prix à imposer son point de vue. Il y eut donc bien une entrée "émotions, affects » dans l'index de notre publication...

Si l'implication experte de Bernard dans l'approche psychanalytique des sociétés le rendait, pour cette raison même, sensible à cet aspect des choses, il n'est pas sûr que la question d'une ethnographie des affects se soit réellement posée ou justifiée à ses yeux ${ }^{1}$. Les occasions d'en reparler ensemble furent malheureusement peu nombreuses et je regrette, compte tenu de son goût profond et rare pour l'ethnographie, de n'avoir pas fait de ce thème un sujet de plus ample discussion entre nous. Des années après son décès, c'est donc naturellement à lui que je pense en abordant cet aspect, et c'est à lui que je dédie ces brefs éléments d'une discussion que nous n'avons pas eue.

Comment approcher ethnographiquement les manières de sentir dans les groupes qu'on étudie ? Comment rendre compte de la prééminence des affects dans les interactions sociales? Une équipe d'anthropologues travaillant sur « l'ethnologie des gens heureux » (Berthon et al., 2009) ${ }^{2}$ m'amena à m'interroger sur le sens du bonheur chez les Sulka de Nouvelle-Bretagne (Papouasie Nouvelle-Guinée). La tendance « corporatiste »-à laquelle je n'ai pas échappéà exclure le bonheur du champ des objets ethnographiables lors de mes terrains successifs fut pour beaucoup dans la difficulté de cet exercice. Cela m'incita du coup à réfléchir à la façon dont les ethnologues traitaient la question du bonheur en particulier et des sentiments en général.

\section{Les dangers de l'émotion}

Dans la culture sulka, le fonctionnement mental et affectif d'une personne est présumé opaque, inconnaissable de celles qui l'entourent. Tandis que l'expression des émotions est relati- vement libre, il apparaît inconvenant de spéculer sur les raisons de ces émotions chez autrui quand elles ne se disent pas ou ne sont pas évidentes. On se refuse donc ouvertement à faire des hypothèses sur ce que pense un individu, pourquoi il le pense, ou ce qui le pousse à se conduire d'une façon plutôt que d'une autre. Les questions de l'ethnologue à ce sujet reçoivent inévitablement le même genre de réponse : «comment savoir ? ", " ça le regarde », " ce sont ses affaires », « lui seul sait... », etc. En s'abstenant ostensiblement d'interpréter un comportement, on se garde d'un jugement implicite qui ferait courir le risque de se trouver partie prenante dans un futur contentieux. Estimer, par exemple, que quelqu'un a agi d'une certaine façon parce qu'il devait probablement avoir honte, ce serait donner à entendre que la honte était plausible, légitime, voire attendue ou prescrite - tout autre sentiment passant alors pour inadéquat ou fautif. De ce point de vue, l'interprétation ne serait pas loin de tenir d'une injonction par défaut plaçant celui qui en fait l'objet en position d'accusé potentiel.

Lorsqu'elle passe pour avoir été intentionnellement induite chez autrui, l'émotion est justiciable d'une procédure de dédommagement matériel. La honte provoquée par une humiliation ou la colère déclenchée par un comportement particulier appellent ainsi la remise d'une réparation en nature ou en argent de la part de celui qui les a causées. Ce rite, que son nom vernaculaire désigne explicitement comme un acte d'apaisement (pet a lep " chasser la honte », rum angaesik "finir la colère" ou dakserai "rompre l'affliction »), ne met pas forcément celui qui s'en acquitte à l'abri de représailles ultérieures. Mais il consacre officiellement la reconnaissance de sa responsabilité individuelle dans le malaise survenu. Il peut d'ailleurs s'agir d'une responsabilité partagée et, dans ce cas, des transactions à l'identique ont lieu entre les parties impliquées, consistant par exemple à échanger exactement la même somme d'argent. Liée à la colère qui en est souvent l'expression, l'affliction, sous sa forme extrême, pouvait autrefois amener le parent d'un défunt à tuer sur-le-champ celui qui lui avait appris la nouvelle $\mathrm{du}$ décès. Induire le chagrin chez quelqu'un, c'était en quelque sorte risquer de s'en rendre coupable par procuration. Aujourd'hui encore, quand elle survient brutalement dans un village, la mort est immédiatement suivie d'une série

1. Convenons qu'il s'agit là d'une impression qui demanderait à être étayée car on sait, par ailleurs, combien cette problématique inspira les approches psychanalytiques des tenants de l'école Culture and personality, ou encore d'auteurs comme John Whiting chez les Kwoma de Nouvelle-Guinée (1941), Erik Erikson ou plus tard Georges Devereux, par exemple.

2. Certaines réflexions présentées dans ce qui suit sont tirées de ma contribution dans ce volume 
d'actes de saccage commentés comme la manifestation d'un désir de meurtre difficilement réfréné : des petits cochons sont lapidés, des volailles tuées, des cultures arrachées et des arbres ou des cocotiers abattus. La sensation éprouvée en de tels moments, disent les Sulka, est celle d'une chaleur intense qui embrase soudainement l'intérieur du ventre " comme un feu ». Tuer, pour reprendre l'observation d'Edward Schieffelin (1977 : 177) à propos des Kaluli de Nouvelle-Guinée constitue le résultat approprié de la « transformation de la douleur en colère »selon une observation relevée ailleurs (Rosaldo, 1984).

Mais les affects que l'on peut qualifier de « négatifs », pour dire vite, n'apparaissent pas seuls en cause. D'un point de vue rituel, les effets de l'admiration, par exemple, ne sont pas traités différemment de ceux d'une offense et appellent eux aussi un dédommagement. Ainsi, et à quelque sentiment qu'il renvoie - joie, ravissement, nostalgie, manque -, l'impact émotionnel induit chez un spectateur par la beauté d'un masque, d'un chant ou d'une danse est également passible d'une procédure de réparation dès que celui qui l'éprouve en fait état (Jeudy-Ballini, 1999). Ce n'est pas là un trait spécifique à l'ethnographie sulka (cf. Dark, 1983; Read, 1955 : 273; Strathern and Strathern, 1971: 126; Schieffelin, 1977 : 143-144 ; Weiner, 1976 : 134135). Dans cette région du monde, en effet, et comme l'observe Simon Harrison à propos des Manambu de Nouvelle-Guinée, l'émotion esthétique est subjectivement perçue comme une agression, une violence, au même titre que la frayeur ou la compassion (1993: 122-125). Chez les Gahuku-Gama de Nouvelle-Guinée, l'impression produite sur un spectateur par un danseur s'exprime conventionnellement comme le fait que celui-ci a «tué » celui-là. Il s'agit, selon Kenneth Read :

«[du] compliment le plus élevé qu'un danseur puisse recevoir, car il y voit à la fois une reconnaissance de sa supériorité sur les autres danseurs et une reconnaissance de ce que ses accomplissements ont gravement déstabilisé ou émotionnellement perturbé son admirateur. » (Read, 1955 : 273)

Notons incidemment, d'ailleurs, qu'on serait tenté de faire le rapprochement avec ce que Morgan Jouvenet écrit du rap et du hip hop en contexte occidental : la performance musicale, souvent comparée à un sport de combat, y est utilisée comme une arme; une arme contre le marché du disque qu'elle cherche à « dynamiter de l'intérieur » (2006: 87), mais également contre les pairs puisqu'elle n'est estimée efficace que si elle " "tue" tout le monde, "atomise la concurrence", la "déchire", réalise un "carnage", etc. ». C'est dans le rap, indique l'auteur, qu'on a commencé à décrire une bonne chanson comme une «tuerie» (2006: 75). Dans les médias - américains notamment -, la rubrique des faits divers avec sa longue liste de rappeurs assassinés par des rivaux montre qu'il ne s'agit pas seulement de mots...

Puissamment intrusive, vécue sur le mode d'une aliénation et d'un préjudice mental, l'emprise émotionnelle en Mélanésie ressortit à un rapport de forces plaçant celui qui se trouve affecté sous le contrôle d'un pouvoir extérieur à lui-même ; en position de vaincu, pour ainsi dire et, plus encore parfois : en situation périlleuse d'y laisser la vie. Les émotions, spécialement quand elles sont fortes ou brutales, font en effet courir le risque d'une dissociation entre l'enveloppe corporelle d'un individu et le double ou principe vital qui l'habite et l'anime. En situation de guerre, c'est cette disjonction - définitive quant à elle - qui s'opère à travers l'acte de tuer et permet au vainqueur de prendre possession du double ou de s'en assurer le contrôle.

«Un homicide, affirme Harrison à propos des Manambu de Nouvelle-Guinée, ne s'entend pas comme l'extermination proprement dite d'une personne mais plutôt comme une forme violente de magie d'amour, une capture ou un transfert de sa force de vie. » (1993 : 122-123)

C'est dire si l'impact esthétique, conçu sur ce modèle d'interaction, constitue une expérience peu anodine. En faisant en sorte de neutraliser cette atteinte à la personne par une compensation matérielle, on évite qu'elle ne donne lieu ultérieurement à un conflit et, en ce sens, on est fondé à penser avec Harrison que les émotions sont autant de "prises permettant à chacun de revendiquer des droits auprès des autres » (1993 : 101).

\section{Le parti pris ethnologique}

Les ethnologues ont en général un faible pour le malheur. Dans la plupart des sociétés qu'ils étudient, les gens heureux n'ont pas d'histoire et le bonheur reste en quelque sorte l'altérité de l'altérité. Si la question du bonheur fut abondamment traitée par la psychologie et la sociologie, elle resta étonnamment négligée par 
l'anthropologie (au moins française) ${ }^{3}$. Certains peuvent même douter que son étude soit pertinente dans des sociétés supposées protégées de l'obsession hédoniste ou du péché de l'individualisme. Vaguement suspecte d'ethnocentrisme, la notion de bonheur s'assimile à une sorte de « trou noir » des monographies, comme si elle était dénuée de réalité objective, de pertinence sociale, et par conséquent de légitimité scientifique en tant qu'objet d'analyse.

Crédité d'une visibilité et d'un degré de sociabilité incomparablement plus forts, le malheur (ou l'idée qu'on en a) inspire bien davantage. À l'instar du méchant des contes ou des westerns qui fait exister l'histoire, c'est par lui, en somme, que s'actualise de préférence le lien social. Et en Mélanésie, de fait, la mort imputée à une attaque de sorcellerie apparaît à première vue autrement plus informative sur un plan ethnographique que celle - combien exceptionnelle pourtant ! - attribuée au grand âge ou au surmenage ${ }^{4}$. C'est d'ailleurs aussi l'avis des Sulka pour lesquels la notion de mort naturelle est le plus souvent dénuée de pertinence ou considérée comme le degré zéro de la culture ${ }^{5}$. On ne peut nier non plus cette réalité anthropologique que dans la plupart des sociétés de cette région du monde, c'est le conflit et la violence, bien davantage que la paix ou l'altruisme, qui organisent la vie sociale. Comme l'observe Simon Harrison,

« Le problème posé par les sociétés mélanésiennes est précisément le degré auquel elles paraissent "socialiser" la violence et l'envisager en soi comme une interaction guère moins "sociale" à part entière que la coopération pacifique. » $(1993: 21)$

La violence et la paix ne sont, de ce point de vue, que les aspects d'une même relation (1993 : 18). Schieffelin écrivait déjà à propos des Kaluli que «ce sont moins les groupes qui produisent des oppositions que les oppositions qui cristallisent les groupes » (1977: 223), considération qu'Harrison généralise en affirmant qu'en Mélanésie «ce sont moins les groupes qui font la guerre que la guerre qui fait les groupes » (1993 : 18).

Au-delà du bonheur, se pose plus largement la question de l'affectivité. Car comprendre une société, c'est connaître les manières de sentir de ses membres, savoir comment elles informent ou organisent leurs interactions sociales, et de quelle façon elles peuvent constituer des modes d'action. Si ce sujet a retenu l'attention d'auteurs ayant travaillé sur l'ethnographie océanienne dans le premier tiers $\mathrm{du} \mathrm{xx}^{\mathrm{e}}$ siècle - à l'instar notamment de Margaret Mead (1928, 1930, 1935), Gregory Bateson (1936) et Reo Fortune (1932) dont on connaît les apports pour l'école culturaliste américaine (Benedict, 1934) -, il s'en faut qu'il ait eu le développement qu'il méritait dans les recherches océanistes des dernières décennies.

Sans doute le désintérêt ethnologique relatif aux affects a-t-il renvoyé en partie à l'idée qu'il n'était pas utile de s'interroger sur des ressentis dont on s'imaginait déjà tout savoir au regard de leur universalité postulée - c'est-à-dire de la réalité substantielle, voire du substrat biologique qu'on leur supposait commun à toutes les sociétés ${ }^{6}$. Ainsi, est-il vraiment besoin d'explorer la notion indigène de douleur ou de peur quand on évoque les épreuves corporelles associées aux rites d'initiation ? Rien n'est moins sûr à en juger par le déficit d'études ethnologiques récentes consacrées à ce type de questions ${ }^{7}$.

Pour schématiser et s'en tenir aux travaux des chercheurs français, il semblerait que l'anthropologie du proche et celle du lointain diffèrent au moins par la place qu'elles accordent à la question des sentiments ${ }^{8}$. En France, de fait, on ne

3. On renvoie ici à l'introduction de l'ouvrage collectif déjà cité (Berthon et al., 2009). A. L. Epstein observe que, « dans le vocabulaire anglais comme dans d'autres langues occidentales, les termes correspondant aux affects négatifs prédominent » (1992: 80).

4. Epstein fait mention du cas d'un Tolai dont la mort fut imputée à l'excès de travail (1992: 99).

5. C'est l'opinion des Sulka à propos de groupes voisins soupçonnés avec mépris d'ignorer la sorcellerie.

6. Considération longtemps mise au compte de l'analogie "naturelle » que certaines expressions faciales des émotions présentaient de manière transculturelle du fait de processus neuromusculaires (Darwin, 1872). Sur cette question éminemment controversée de l'universalité des émotions, on se contentera de renvoyer aux nombreux travaux de Paul Ekman reprenant notamment le débat engagé entre les relativistes culturels comme Margaret Mead et les darwiniens qui défendent l'idée d'expressions émotionnelles biologiquement déterminées et communes à l'humanité.

7. On notera toutefois que la question des émotions fait partie du champ traditionnel des études religieuses. Le fondement émotionnel des comportements rituels (Lutz, 1988 ; Lutz and White, 1986), ou le rôle des facteurs émotionnels dans les processus de mémorisation et de transmission des pratiques religieuses (voir les travaux de Pascale Bonnemère [2008] ou de Harvey Whitehouse [1995 et 2000]), par exemple, ont fait depuis longtemps l'objet d'approches anthropologiques. Mais ce n'est pas, on l'aura compris, le sujet qui nous retient ici et qui a trait plutôt à la question de savoir ce qui fait émotion ou ce qu'est une émotion dans une société donnée.

8. Notons qu'on serait tenté de faire la même remarque à propos de la différence entre l'anthropologie française et l'anthropologie britannique ou américaine. On le constate tout au long de ce texte au nombre des citations d'origine anglo-saxonne : à l'exception d'auteurs anciens (plus souvent sociologues) ou de quelques chercheurs contemporains ( $c f$. infra F. Laplantine et A. Surrallés), les ethnologues français travaillant sur la question des affects à partir de leur propre ethnographie de terrain restent encore rares. 
saurait se livrer à une ethnologie de la vieillesse sans évoquer le sentiment de solitude, d'abandon, de dépression ou de détresse qui est souvent le quotidien des vieux. De la même façon, entreprendre une ethnographie des banlieues « difficiles », n'impliquerait pas seulement de porter attention au chômage ou à la précarité matérielle, mais aux sentiments de frustration, d'injustice, d'humiliation ou de colère qui rendent le quotidien problématique et font de l'action collective un moment d'exaltation. Dans ces contextes, penser l'économique et le social c'est nécessairement mobiliser la dimension affective qui les constitue.

En Océanie, à l'inverse, on peut étudier d'importants aspects de la vie sociale (les mythes, les activités de subsistance, les rites d'initiation, etc.), sans que l'étude des sentiments qui les fondent ou les nourrissent s'impose comme une nécessité. A. L. Epstein, spécialiste des Tolai de Nouvelle-Bretagne - et qui interpréta certaines de ses données ethnographiques à la lumière de la théorie psychanalytique ${ }^{9}$-, observe ainsi qu'en anthropologie le rapport au travail n'a le plus souvent été approché qu'en termes économiques, ce qui, écrit-il, impose de sérieuses limites à notre compréhension de sa nature et de son rôle dans les sociétés étudiées (1992: 82). Selon la manière dont ils s'équilibrent ou non, par exemple, l'esprit de compétition et la prégnance du sentiment d'envie dans maintes sociétés mélanésiennes (voir Kahn, 1986) peuvent rendre compte de l'émulation qui résulte du désir de se distinguer - comme chez les Tolai - ou au contraire des retenues que s'imposent à euxmêmes les hommes dans leurs ambitions. Les Sulka, par exemple, attribuent à la réussite personnelle une manière dangereuse de s'exposer. Des décès imputés à de la sorcellerie ont ainsi leur origine supposée dans la jalousie suscitée par certains accomplissements, réels ou revendiqués, comme si la popularité qu'ils valaient à leurs auteurs devait porter ombrage aux capacités des autres membres de la communauté. Cette jalousie omniprésente est supposée inhiber (ou dissuader de) tout esprit d'entreprise (JeudyBallini, 2004 : 131-133). Un villageois déclarait :

« Les gens ont peur et ne montrent pas trop de quoi ils sont capables. Parce que si tu vas de l'avant, tu vas complètement [= tu meurs] ! Si tu travailles à quelque chose, que cela devient quelque chose de bien qui te vaut le respect de ton nom, un autre dira : "Ah, cet homme-là il aurait donc des bras et pas nous! Qu'on s'en débarrasse, il nous rabaisse !" Et c'est comme ça qu'on meurt! Tu tires ta mort avec ton savoir. Si tu travailles et que tu montres de quoi tu es capable, la mort te prend. » (Extrait d'entretien, 1994)

On dit aussi qu'un des moyens les plus sûrs de nuire à une personne est d'en dire constamment du bien : d'en louer les qualités, d'en exalter les mérites, d'en prononcer souvent le nom, de la créditer de compétences supérieures, de la citer en exemple, de faire référence à son autorité, de se réclamer de son savoir, de sa protection. Dans l'ancien système de chefferie, une sorte de garde rapprochée (humevek) entourait en permanence les "grands hommes » (taven) et les "grandes femmes » (kheng) pour assurer leur protection car ils étaient, plus que tous autres dit-on, une cible privilégiée des attaques de sorcellerie.

À Avatip, où la guerre constitue un mode de socialité intense, les interactions entre les combattants ne sont possibles, explique Harrison, qu'à condition qu'ils fassent en sorte de suspendre tout affect, y compris l'animosité. « Les hommes nient ressentir de la colère précisément parce qu'ils la tiennent pour un type de lien affectif », écrit-il (1993 : 101).

« Éprouver de la colère ou de l'hostilité envers leurs adversaires équivaudrait pour eux à admettre un état de faiblesse et de dépendance antithétique des valeurs masculines avatip; ce serait reconnaître tacitement qu'ils sont restés sous l'emprise du pouvoir de l'ennemi. » (Harrison, 1993 : 101)

Acquise au terme d'une magie d'insensibilisation, la capacité d'agression suppose donc une impassibilité, un retrait de tout lien de réciprocité émotionnelle vis-à-vis des autres. Cette forme d'immunisation quasiment autistique se veut la manifestation d'un pouvoir transcendant les subjectivités individuelles (1993: 111-112).

\section{La compétence émotionnelle}

Outre l'héritage probable de la pensée structuraliste - qui a laissé à distance les émotions comme un hors-sujet anthropologique ou les a rabattues sur du psychologisme, voire du biologisme (Lévi-Strauss, 1971 : 577) -, l'idée convenue de sociétés non individualistes, attachées au respect de la tradition et au sein desquelles les comportements supposeraient une forte dose de normalisation collective, tend sans

9. Il s'intéressa par exemple à l'analyse des rêves (Epstein, 1990) ou montra encore comment l'ethos paranoïde des Tolai manifeste dans la méfiance et la suspicion imprégnant tout rapport interpersonnel au sein de cette population - se répercutait dans l'organisation locale et d'autres faits sociaux (Epstein, 2000). 
doute à expliquer la minoration relative de l'affectivité dans l'approche ethnologique des ailleurs ${ }^{10}$. Pourtant, c'est une évidence de considérer que l'allégeance aux normes n'implique pas l'éradication ou l'anesthésie des affects individuels. Chez les Sulka, dont la vie s'organise autour d'échanges incessants et d'une idéologie de la réciprocité, existe une réticence profonde à se séparer de ce qu'on a. Le fait que les échanges ressortissent à des conventions sociales et que les biens n'acquièrent de valeur qu'en tant qu'ils circulent, ne doit pas occulter pour autant la détestation que cela suscite sur un plan personnel et les stratégies clandestines mises au point pour se soustraire aussi souvent que possible à tout partage.

Marcel Granet (in Le Breton, 2006 : 334) récusait l'idée que la codification des comportements rituels puisse altérer la sincérité et la spontanéité des émotions. À propos des pleurs, lamentations et autres expressions spectaculaires de chagrin ayant cours lors des rituels funéraires, Marcel Mauss observait de son côté :

«Ces cris, ce sont comme des phrases et des mots. Il faut les dire, mais s'il faut les dire c'est parce que tout le groupe les comprend. On fait donc plus que de manifester ses sentiments, on les manifeste aux autres puisqu'il faut les leur manifester. On se les manifeste à soi en les exprimant aux autres et pour le compte des autres. » (Mauss, 1968-1969 : 88, in Le Breton, 2006 : 334)

\section{Ainsi, et dans les termes de Le Breton :}

«Pour qu'une émotion soit ressentie, perçue et exprimée par l'individu, elle doit appartenir sous une forme ou sous une autre au répertoire culturel de son groupe. [...] Une culture affective est socialement à l'œuvre. Chacun impose sa coloration personnelle au rôle qu'il joue avec sincérité ou distance, mais un canevas demeure qui rend les attitudes reconnaissables. » (2006 : 335)

Reconnaissables, c'est-à-dire propres à satisfaire le désir d'adhésion sociale de celui qui en fait montre"1. L'acquisition de cette " compétence émotionnelle » (Lutz and White, 1986) est précisément l'une des composantes fondamentales de la socialisation, ainsi que Radcliffe-Brown (1922) l'avait montré en son temps.

Les conditions matérielles de vie dans une communauté, les relations entre ses membres et l'imaginaire local à la fois génèrent des théories particulières de l'émotion et sont régis par elles. En ce sens, et comme le suggère l'étude transculturelle de Russell (1991), une approche anthropologique devrait porter attention non seulement au contenu de l'émotion en soi mais également à ce qui l'induit, au statut de ceux qui peuvent ou non l'éprouver et peuvent ou non en être la cible ${ }^{12}$, à la façon dont elle s'exprime propre à définir un mode d'action -, ou encore à ses effets. On sait par exemple que les Ifaluk de Micronésie, qui n'ont pas de terme générique pour désigner la peur, divisent celle-ci en plusieurs catégories selon (entre autres critères) ce qui la cause et le moment où elle est ressentie. Assimilée à un signe de maturité sociale, la capacité à avoir peur joue un rôle dans le système organisant les rapports de pouvoir et l'allégeance aux normes morales de la vie communautaire. C'est la peur (notamment peur anticipée de la colère des autres et peur des esprits) qui commande le respect des interdits.

« Les sociétés, en infère Lutz, diffèrent quant au degré auquel elles perçoivent le danger et quant à la nature de ce qui constitue à leurs yeux un danger. " (1988: 183)

Mais si une telle perception varie au cours du temps, elle varie également, à une époque donnée, au sein d'une même société. Par exemple, tandis qu'une conception à peu près consensuelle du danger prévaut dans les diverses localités sulka, le rapport différentiel à la peur définit quant à lui des postures ou des positionnements sociaux spécifiques à visée clairement démarcative (Jeudy-Ballini, 2002). Ainsi, les représentations courantes qui font de la peur un facteur de risque ne dissocient pas l'occurrence d'un malheur de l'émotion préalable de celui

10. Notons qu'il existe quantité d'autres raisons qui ont fait l'objet d'analyses dans la plupart des travaux traitant de la question des émotions, des affects ou du sensible (voir par exemple Epstein, 1992 ; Surrallés, 2003 et 2004 ; Beatty, 2005 ; Laplantine, 2005 ; Berthon et al., 2009).

11. À propos d'une étude sur les rites de deuil des Amérindiens du Brésil, Mossière observe que : «À travers l'activité rituelle, l'individu signale en effet une forme de tristesse qui est émise selon une forme jugée appropriée par les autres membres du groupe, il indique par là son adhésion aux normes collectives, ce qui permet son acceptabilité sociale. L'expression de l'émotion représente donc un moyen de contrôler l'identité signalée au groupe ; en ce sens, l'affect devient méta-affect car la tristesse commente le désir d'acceptabilité sociale » (Mossière, $2004: 53$ ).

12. «La place assurée des chefs à la tête de la structure sociale ifaluk est à la fois démontrée et confortée par l'incapacité de leurs sujets à invoquer une colère justifiée à leur égard », écrit Lutz (1988:170). L'auteur observe plus loin : « La colère justifiée est une pratique idéologique liée à l'exercice du pouvoir. Plus une faction est puissante dans la société ifaluk - celle des chefs, des frères, des femmes âgées d'une maisonnée - plus elle fait un usage étendu de ce concept et plus sa légitimité et son influence s'en trouvent renforcées » (Lutz, $1988: 182)$. 
qu'il affecte. Pour les Sulka en effet, et indépendamment de toute affiliation religieuse, il est de notoriété que les esprits, à l'instar des animaux dangereux comme les crocodiles ou les requins, agressent de préférence ceux qui les redoutent. À l'inverse, l'incroyance des hommes, ou plus exactement leur volonté de ne pas céder à la peur, ôte prise aux esprits ou aux animaux menaçants et les réduit à l'impuissance. En bref et pour l'exprimer dans les termes d'un homme se revendiquant catholique :

« Tant qu'on ne sait pas qu'un endroit est dangereux, on y est en sûreté. » (Extrait d'entretien, 1994)

De nos jours, ce point de vue est systématisé par les nouveaux convertis à l'Église des Adventistes du septième jour aux yeux desquels les esprits n'existent que dans la mesure où on a la faiblesse de croire en eux. S'imposer de ne pas y croire, c'est refuser de céder à la peur et les contraindre de ce fait à rester inoffensifs. Les Adventistes nouvellement convertis assignent ainsi une valeur expérimentale et démonstrative aux actes anti-conformistes à travers lesquels ils tentent - publiquement ou clandestinement - de surmonter leurs propres peurs et d'éprouver le pouvoir de leur incroyance : par exemple en traversant volontairement un endroit réputé peuplé d'esprits malfaisants ou en transgressant intentionnellement des interdits coutumiers. Bien évidemment, et malgré leur désir de se différencier des catholiques qu'ils critiquent de manière virulente, les Adventistes sulka ne mettent jamais en cause la réalité du pouvoir des esprits ou de la magie. À l'instar de l'Église fondamentaliste de la New Tribes Mission décrite par Andrew Lattas, le mouvement adventiste sulka tire en effet sa force de « la réalité qu'il accorde au mal » (1998: 313). Il faut en somme que le mal existe pour que l'Église puisse démontrer qu'elle en protège, et il faut que le mal continue à faire peur aux uns pour que les autres puissent se prévaloir de la supériorité protectrice de leur nouvelle religion. Le contrôle des émotions est un enjeu stratégique et, aux yeux de ceux qui y travaillent, un enjeu vital.

\section{L'émotion, un objet anthropologique en soi ?}

Les villageois sulka éprouvent-ils des formes d'angoisse existentielle, de mélancolie ou d'ennui ? Comment vivent-ils la transparence imposée par la vie en communauté ? D'un point de vue affectif, ont-ils intégré de façon commune à tous l'impossibilité de la fuir? Le désir de solitude a-t-il un sens? Comment un homme parvient-il à supporter le voisinage quotidien d'un individu dont il pense être la cible de la sorcellerie? Y a-t-il des sentiments dépourvus d'existence nominale (tout comme certaines couleurs)...? Cette dernière question est spécialement complexe, comme le remarque Yannick Jaffré :

"puisqu'en ce domaine "affectif", le signe et le référent vont parfois jusqu'à se confondre, au point que l'on puisse se demander si certains sentiments seraient éprouvés s'ils n'étaient préalablement nommés. » $(2006: 6)^{13}$

Quoi qu'il en soit, et s'agissant des Sulka que j'ai connus, ces questions et bien d'autres restent toujours sans réponse pour moi.

De telles interrogations, qui renvoient aux manières individuelles ou communes de sentir et par conséquent de vivre les relations sociales, ne sauraient se réduire à une anthropologie des émotions dans la mesure où l'on ignore a priori ce qui se définit comme « émotion » au sein des sociétés qu'on se propose d'étudier - cette notion n'ayant parfois même pas d'équivalent dans la langue vernaculaire ${ }^{14}$. A l'instar de ce que François Laplantine écrit des sensations, les émotions sont éminemment fluctuantes :

« Elles n'ont pas, contrairement au politique, à proprement parler de consistance. Elles ne sont pas substantielles et donc pas substantialisables. Elles répugnent à la généralisation à la hauteur du concept et forment encore moins des essences. [...] Mais c'est précisément cette inessentialité qui est la réalité. » (2005: 153-154)

Le fait que la même société qui n'a pas de mot correspondant au concept d'"émotion" dispose en revanche de quarante-six termes distincts pour désigner la colère (Russell, 1991 : 429) atteste la nécessité démontrée par Laplantine de penser le divers et la nuance. De fait, comme ce dernier l'observe :

« [...] il n'existe pas de couleur en soi mais toute une gamme de couleurs pouvant osciller entre différents tons. » (Laplantine, 2005 : 153-154)

En outre, pour rester dans la comparaison, comment décider d'un «ton » plutôt que d'un

13. On pense ici à la citation de La Rochefoucauld rapportée par Le Breton : « Il y a des gens qui n'auraient jamais été amoureux s'ils n'avaient jamais entendu parler de l'amour » (2006:335).

14. Russell (1991: 429) signale ainsi que ni les Tahitiens (Levy, 1973: 271), ni les Bimin-Kuskusmin de Papouasie Nouvelle-Guinée, ni les Gidjingali d'Australie, les Ifaluk de Micronésie ou les Samoans par exemple, n'ont de mot générique pour « émotion » ou « sentiment »- ce qui ne met évidemment pas en cause, faut-il le préciser, l'existence de la notion. 
autre? Quand les Tolai usent ainsi d'un même mot pour signifier "déçu » et "paresseux » (Epstein, 1992 : 68), en quoi serait-on plus fondé à mobiliser une anthropologie des émotions qu'une anthropologie du travail ?

On sait par ailleurs que maintes cultures n'établissent pas d'opposition sémantiquement pertinente entre sentiments et sensations physiques parfois réunis sous un vocable identique ainsi qu'il est d'usage à Samoa (Russell, 1991 : 429). En Nouvelle-Bretagne, les Baining associent la faim au sentiment de solitude (Fajans, 1997 : 119), à l'instar des Wamira de Nouvelle-Guinée pour lesquels la faim relève d'une condition émotionnelle ou sociale plutôt qu'organique (Kahn, 1986 : 39). Chez les Sulka, la douleur induite par un deuil ou la séparation d'avec un proche induit une sensation d'épuisement et d'intense faiblesse qui se traduit, dans le comportement des gens affectés, par le renoncement provisoire à la mobilité. Une telle corrélation est également attestée dans la société tahitienne où n'existe pas de mot équivalent à celui de «tristesse ». Selon Lévy (in Russell, $1991: 429$ et 431), les notions de fatigue, de nostalgie, de solitude ou de dépression par lesquelles peut se dire la tristesse s'assimilent moins à des affects qu'à une maladie organique.

L'absence d'opposition sémantique est aussi attestée dans bien des cas entre émotion et cognition, sentir et connaître, intelligible et sensible (Lutz, $1983: 251$; $1985: 46-47$ ), comme chez les Manambu où l'on désigne par un même terme, mawul, à la fois le siège des émotions et celui de la connaissance (Harrison, 1993: 97). Pareilles indifférenciations se retrouvent d'ailleurs dans maintes cultures non océaniennes, par exemple dans la plupart des sociétés amazoniennes (Laplantine, 2005: 11). Ainsi, écrit Alexandre Surrallés, les Candoshi du Pérou, qui perçoivent la maladie ou la mort comme des affects, ne distinguent pas entre aimer et penser (2003:66). Et c'est aussi en renonçant à dissocier ce qui n'a pas lieu de l'être, l'affect et la connaissance, que l'on est le plus à même de comprendre la portée heuristique de l'usage des hallucinogènes chez les Mazatèques du Mexique selon Magali Demanget (2009). Notons qu'il ne s'agit pas là d'un trait exclusif aux sociétés «tradition- nelles ». On peut en effet le retrouver au sein de notre propre société, même si la "dichotomie tyrannique » dénoncée par Goodman (1990 : 290) entre émotion et raison y reste généralement prégnante ${ }^{15}$. Ainsi qu'on le montrait dans une étude ethnographique sur le rapport à l'objet parmi les collectionneurs français d'art primitif (Derlon et Jeudy-Ballini, 2008), l'expérience esthétique se joue du clivage entre le cognitif et l'émotif pour devenir en soi un mode de connaissance, comme il ressort aussi d'autres formes d'investissement affectif intense avec des objets.

Il est intéressant de noter que ce type d'observations rejoint celles mises en évidence par les biologistes dans le système neurocérébral humain où la capacité à ressentir des émotions fait partie intégrante des procédures de raisonnement et de prise de décision (Damasio, 1995). Aussi doit-on admettre, selon la formulation de Jean-Didier Vincent, spécialiste de la chimie du cerveau, qu'il n'existe " en aucun moment du sujet pur, mais toujours un sujet ému, affecté par le monde et par le corps" (Le Monde, 9/7/2004) ${ }^{16}$. Parce qu' ' un homme qui pense est un homme affecté » (Le Breton, $2006: 334$ ) et que les émotions n'ont pas de réalité en dehors de la pensée - ce sont des "pensées incorporées », dans les termes de M. Rosaldo (1984: 143) -, l'expression « anthropologie des émotions » qui donnerait à penser que l'anthropologie puisse traiter de « sujets purs » apparaît inadaptée.

« L'émotion est un aspect inhérent à toute activité, à toute interaction, à toute représentation, à toute production et reconnaissance du sens. Il s'ensuit que ne peut exister, à proprement parler, une anthropologie qui s'intéresse à l'affectivité comme à un quelconque autre domaine. [...] l'affectivité doit participer de l'explanatio - le moyen qu'on se donne pour comprendre - et pas du seul explanandum - ce qu'il faut expliquer. Dans les études du fait politique, de la parenté ou des rapports à l'environnement par exemple, elle est non seulement une instance du social, mais elle fait partie de son approche. » (Surrallés, 2004 : 62)

L'intégrer dans toute approche anthropologique, précisément, devrait tenir lieu de programme. Or la tâche est difficile, qui invite à reconsidérer, outre nos manières de penser, nos

15. À cet égard, il est notable que pour se défendre des critiques l'accusant de méconnaitre l'importance de la vie affective dans l'ethnographie des sociétés, Claude Lévi-Strauss ne trouve meilleur moyen que de hiérarchiser entre affects et intellect : « [...] il est vrai que je m'attache à discerner, derrière les manifestations de la vie affective, l'effet indirect d'altérations survenues dans le cours normal des opérations de l'intellect [...]. Car ce sont ces opérations seules que nous pouvons prétendre expliquer, parce qu'elles participent de la même nature intellectuelle que l'activité qui s'exerce à les comprendre. [...] Toute manifestation de la vie affective qui ne refléterait pas, sur le plan de la conscience, quelque incident notable bloquant ou accélérant le travail de l'entendement ne relèverait plus des sciences humaines ; elle serait du ressort de la biologie [...] » (Lévi-Strauss, 1971 : 596-597).

16. «C'est pourquoi, poursuit J.-D. Vincent, l'affect pour moi possède un sens très large : c'est tout ce que le cerveau sait du corps et, par l'intermédiaire du corps, tout ce qu'il sait du monde » (Le Monde, 9/7/2004). 
propres attentes épistémologiques. De fait, comme l'écrit François Laplantine à propos de notre discipline :

«Ce qui est privilégié, c'est l’ordre de la raison faisant apparaître une intelligibilité cachée derrière un désordre apparent de sensations et d'émotions. Tout se passe comme si, dans la polysémie du mot sens désignant à la fois le sensible et le sensé, la sensation et la signification, le rationalisme était amené à trancher et à subordonner. Ce que Montaigne appelle l'univers "ondoyant et divers" de la sensibilité est non seulement organisé, mais risque d'être neutralisé, voire disqualifié. Bref, il y a bien, dans l'une des traditions de la pensée européenne (et seulement dans cette tradition que l'on ne rencontre dans aucune autre société), un conflit - censé être résolu par une hiérarchisation entre la multiplicité du sensible et l'universalité de l'intelligible, entre le corps et la pensée, et notamment la pensée sociale et politique. » $(2005: 100)$

Il y a là une incitation même à repenser profondément les cadres et la visée de l'anthropologie selon cette « épistémologie de la continuité » prônée par Laplantine, qui devrait permettre de :

« penser ensemble des domaines le plus souvent considérés comme séparés : l'esthétique, le politique, l'éthique et l'histoire. Un tel mode de connaissance qui engage la totalité de l'affectivité et de l'intelligence, s'oppose aux césures en série de la raison et de l'émotion, du sens et du son, du contenu et de la forme, de l'Occident et de l'Orient [...]. » (2005: 155)

Où l'on voit que l'anthropologie, dont on a parfois prédit la fin avec la disparition supposée de ses sujets d'étude, n'est pas au bout de sa tâche...

\section{BIBLIOGRAPHIE}

BAteson Gregory, 1936. Naven: A Survey of the Problems Suggested by a Composite Picture of the Culture of a New Guinea Tribe Drawn From Three Points of View, Cambridge, Cambridge University Press.

BEATTY Andrew, 2005. Emotions in the field: what are we talking about?, Journal of the Royal Anthropological Institute (N.S.) 11, pp. 11-37.

BENEDICT Ruth, 1934. Patterns of culture, Boston and New York, Houghton Mifflin.

Berthon Salomé, Sabine Chatelain, Marie-Noëlle Ottavi et Olivier Wathelet (eds), 2009. Ethnologie des gens heureux, Paris, Éditions de la maison des sciences de l'homme.

BonnemÈre Pascale, 2008. Du corps au lien : l'implication des mères dans les initiations masculines des Ankave-Anga, in I. Thery et P. Bonnemère (éds), Ce que le genre fait aux personnes, Paris, Éditions de l'EHESS, pp. 75-90.

Damasio Antonio R., 1995. L'Erreur de Descartes, Paris, Odile Jacob.

DARK Philip, 1983. Among the Kilenge Art is "something which is well done", in Sidney M. Mead and Bernie Kernot (eds), Art and artists of Oceania Palmerston North, The Dunmore Press, pp. 25-44.

DARWIN Charles, 1872. The Expression of the Emotions in Man and Animals, London, John Murray.

Demanget Magali, 2009. Chamanisme, ethnologie et tourisme : le partage du bonheur en question, in Salomé Berthon, Sabine Chatelain, Marie-Noëlle Ottavi et Olivier Wathelet (éds), Ethnologie des gens heureux, Paris, Éditions de la maison des sciences de l'homme, pp. 81-95.

Derlon Brigitte et Monique Jeudy-Ballini, 2008. La Passion de l'art primitif. Enquête sur les collectionneurs, Paris, Gallimard.

Ekman Paul, 1973. Darwin and Facial Expression: $A$ Century of Research in Review, New York, Academic Press Inc, pp. 169-222.

-, 1980. The Face of Man: Expressions of Universal Emotions in a New Guinea Village, New York, Garland STPM Press.

EpsteIn Arnold L., 1990. Dream Drawings, Affect, and Cultural Themes: Pointers from Some Tolai Data, in L. Bryce Boyer and Simon A. Grolnick (eds), The Psychoanalytic Study of Society 15, Hillsdale, NJ, the Analytic Press, pp. 179-204.

-, 1992. In the Midst of Life. Affect and Ideation in the World of the Tolai, Berkeley and Los Angeles, University of California Press.

_, 2000. The paranoid ethos in Melanesia: The case of the Tolai, Journal de la Société des Océanistes 110 (1), pp. 3-18

FAJAns Jane, 1997. They Make Themselves. Work and Play among the Baining of Papua New Guinea, Chicago and London, The University of Chicago Press.

FortunE Reo, 1932. Sorcerers of Dobu, London, Routledge and Kegan Paul.

GoOdman Nelson, 1990 (1968). Langages de l'art. Une approche de la théorie des symboles, Paris, Jacqueline Chambon.

HARRISON Simon, 1993. The Mask of war. Violence, ritual and the self in Melanesia, Manchester and New York, Manchester University Press.

JAFFRÉ Yannick, 2006. Les terrains d'une anthropologie comparative des sensibilités et des catégories affectives, Face à Face. Regards sur la santé 9 : Émotions, corps et santé. Un gouvernement par la parole?, Bordeaux, université de Bordeaux II (revue en ligne http://www.ssd.u-bordeaux2.fr/faf/ archives/index.htm).

Jeudy-Ballini Monique, 1999. «Dédommager le désir ». Le prix de l'émotion en Nouvelle-Bretagne, Terrain 32, pp. 5-20. 
—, 2002. Le christianisme revisité ou le meilleur de la tradition, in Christine Hamelin et Éric Wittersheim (éds), La tradition et l'État. Églises, pouvoirs et politiques culturelles dans le Pacifique, Paris, L'Harmattan, pp. 59-81.

_, 2004. L'art des échanges. Penser le lien social chez les Sulka, Lausanne, Éditions Payot.

_, 2009. L'air du bonheur dans la société sulka, in Salomé Berthon, Sabine Chatelain, Marie-Noëlle Ottavi et Olivier Wathelet (éds), Ethnologie des gens heureux, Paris, Éditions de la maison des sciences de l'homme, pp. 149-160.

Jouvenet Morgan, 2006. Rap, techno, électro... Le musicien entre travail artistique et critique sociale, Paris, Éditions de la maison des sciences de l'homme.

Kahn Miriam, 1986. Always Hungry, Never Greedy. Food and the Expression of Gender in a Melanesian Society, Cambridge, Cambridge University Press.

LAPLANTINE François, 2005. Le social et le sensible. Introduction à une anthropologie modale, Paris, Téraèdre.

Lattas Andrew, 1998. Cultures of Secrecy. Reinventing Race in Bush Kaliai Cargo Cults, Madison, The University of Wisconsin Press.

Le Breton David, 2006. Émotion, in Michela Marzano (éd.), Le Dictionnaire du corps, Paris, PUF, pp. 333-337.

LÉVI-Strauss Claude, 1971. Les Mythologiques, tome IV : L'Homme nu, Paris, Plon.

LeVy Robert I., 1973. Tahitians, Chicago, University of Chicago Press.

Lutz Catherine, 1983. Parental Goals, Ethnopsychology, and the Development of Emotional Meaning, Ethos 11 (4), pp. 246-262.

-, 1985. Ethnopsychology Compared to What? Explaining Behavior and Consciousness among the Ifaluk, in Geoffrey M. White and John Kirkpatrick (eds), Person, Self, and Experience: Exploring Pacific Ethnopsychologies, Berkeley, University of California Press, pp. 35-79.

—, 1988. Unnatural Emotions. Everyday Sentiments on a Micronesian Atoll and Their Challenge to Western Theory, Chicago and London, The University of Chicago Press.

Lutz Catherine and Geoffrey White, 1986. The anthropology of emotions, Annual Review of Anthropology 15, pp. 405-436.

MEAD Margaret, 1928. Coming of age in Samoa. A psychological study of primitive youth for Western civilization, New York, William Morrow.

_, 1930. Growing up in New Guinea. A comparative study of primitive education, New York, William Morrow.
—, 1935. Sex and temperament in three primitive societies, New York, William Morrow.

MossiÈre Géraldine, 2004. Expressivité rituelle : Corps et discours dans le culte dominical d'une église d'immigrants à Montréal, mémoire de mâ̂trise ès-sciences en anthropologie, Montréal, université de Montréal, $168 \mathrm{p}$.

RadCLIFFE-Brown Alfred R., I922. The Andaman Islanders, Glencoe (Illinois), The Free Press.

READ Kenneth E., 1955. Morality and the Concept of the Person among the Gahuku-Gama, Oceania 25 (4), pp. 233-282.

RosALDo Michelle, 1984. Toward an Anthropology of Self and Feeling, in R. A. Shweder and R. A. LeVine (eds), Culture Theory: Essays on Mind, Self, and Emotion, Cambridge, Cambridge University Press.

Rosaldo Renato I., 1984. Grief and a Headhunter's Rage: On the Cultural Force of Emotions, in $\mathrm{S}$. Plattner and E. M. Bruner (eds), Text, play, and story: The construction and reconstruction of self and society, Washington DC, American Ethnological Society, pp. 178-195.

Russell James A., 1991. Culture and the Categorization of Emotions, Psychological Bulletin 110 (3), pp. 426-450.

SCHIEFFELIN Edward L., 1977. The Sorrow of the Lonely and the Burning of the Dancers, St Lucia, University of Queensland Press.

Strathern Andrew and Marilyn Strathern, 1971. Self-decoration in Mount Hagen. Toronto and Buffalo, University of Toronto Press.

Surrallés Alexandre, 2003. Au cour du sens : perception, affectivité, action chez les Candoshi, Paris, CNRS Éditions/Éditions de la maison des sciences de l'homme.

—, 2004. Des états d'âme aux états de fait. La perception entre le corps et les affects, in Françoise Héritier et Margarita Xanthakou (éds), Corps et affects, Paris, Odile Jacob, pp. 59-75.

VINCENT Jean-Didier, 2003. Le cæur des autres. Une biologie de la compassion. Paris, Plon.

WeIner Annette, 1976. Women of Value, Men of Renown. New Perspectives in Trobriand Exchange, Austin and London, University of Texas Press.

Whitehouse Harvey, 1995. Inside the Cult. Religious Innovation and Transmission in Papua New Guinea, Oxford, Clarendon Press.

—, 2000. Arguments and icons: Divergent modes of religiosity, Oxford, Oxford University Press.

Whiting John W. M., 1941. Becoming a Kwoma: Teaching and Learning in a New Guinea Tribe, New Haven, Yale University Press. 